\title{
An Artificial intelligence approach to detection of high impedance fault
}

\author{
Rakesh Kumar ${ }^{1}$, Neelam Saini ${ }^{2}$, Ankita Saini ${ }^{3}$ \\ Assistant Professor Department of ECE, JMIETI, Radaur, India ${ }^{1}$ \\ M.Tech Scholar, D.C.R.University of Science \& Technology, Murthal (Sonepat),Haryana, India ${ }^{2,3}$
}

\begin{abstract}
This paper presents a new method to detect high impedance faults in radial distribution systems. Magnitudes of third and fifth harmonic components of voltages and currents are used as a feature vector for fault discrimination. The proposed methodology uses a back propagation neural network as a classifier for identifying high impedance arctype faults. The network learns from the data obtained from simulation of a simple radial system under different fault and system conditions. Compared to a feed-forward neural network, a properly tuned back propagation network gives quicker response.
\end{abstract}

Keywords: Fault identification, distribution networks, high-impedance arc-faults, feature vector, back-propagation network.

\section{INTRODUCTION}

HIGH impedance faults (HIFs) on distribution systems can create unique challenges for the protection engineer. HIFs that occur do not produce enough fault current detectable by conventional over-current relays or fuses [1]. A high impedance ground fault results when a primary conductor makes unwanted electrical contact with a road surface, sidewalk, tree limb, or with some other surface, or object which restricts the flow of fault current to a level below that reliably detectable by conventional over-current devices [1]. These faults are characterized by intermittent arc-type nature and very low current rich in low harmonic content and high frequency noise spectra. The failure of HIF detection may lead to potential hazards to human beings and potential fire [2]. Therefore, from both public safety and operational reliability viewpoints, detection of HIFs is critically important. Engineering efforts for the development of a reliable method for the detection of high impedance arc-type faults led during the last two decades to important progress in understanding the electrical characteristics of these faults and in the evaluation of several detection concepts [3]. Various techniques of fault detection encompass fractal techniques [4], expert systems [5], neural networks [6-8] and dominant harmonic vectors $[9,10]$

The use of high frequency harmonics is not feasible in practical relay because of the filtering by the substation current transformers. Other methods that try to reduce the limitation of frequency domain methods include Kalman filtering [11] and wavelet transform based methods [2, 12]. Among many techniques proposed by different research groups, use of information contained in the low frequency spectral behavior, in terms of both magnitude and phase, seems to be the most promising approach for the next steps which will bring the industry closer to the realization of a fully operation HIF detector [13]. In [14], a novel time domain HIF detection scheme based on low frequency harmonic excursion patterns and phase portraits is used to detect the high impedance arc-type faults on a radial distribution system. In this paper we propose a novel approach of employing back propagation neural networks to detect HIFs in radial distribution system. In the proposed scheme, the reference vectors are set to the locations mostly matching the probability distributions of training to improve the characteristics of back-propagation. The improved characteristics ensure more accurate classification results. The sample system studied in this paper is a $25 \mathrm{kV}$ power distribution network studied in [14]. The paper is organized as follows. Section 2 deals with a brief review of the system description and fault simulation method.

\section{SYSTEM DESCRIPTION AND FAULT SIMULATION}

Single line diagram of the sample radial distribution system [14] is shown in Fig. 1. The distribution line is represented by lumped passive elements without mutual coupling. Constant impedance load is assumed. Fig. 2 represents the equivalent per phase circuit during fault with nonlinear arcing fault resistance $R f$. The training data is obtained by applying nonlinear and linear faults to the circuit model at different fault locations $x$. The system parameters and nonlinear arc model are given in the Appendix. Using Fig. 2, following nodal equations can be written in s-domain. The variables in s-domain are shown in capital letters. For linear faults, the fault resistance is not varying as shown in Appendix.

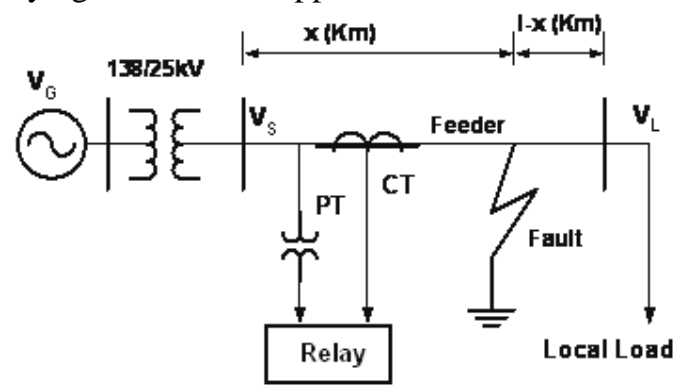

Fig. 1 Single line diagram of a radial distribution line 


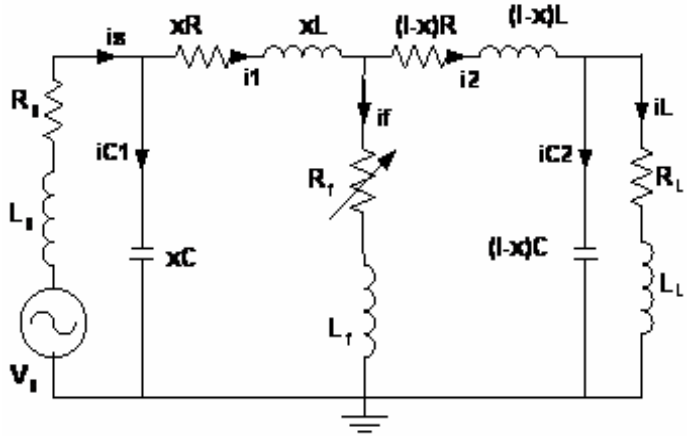

Fig. 2 per phase equivalent circuit with HIF arc-type fault

$$
\begin{aligned}
& I_{S}=\left(V_{S}-V_{1}\right) \frac{1}{L_{S} s+R_{S}} \\
& I_{C 1}=I_{S}-I_{1} \\
& V_{1}=\frac{1}{x C s} I_{C 1} \\
& I_{1}=\left(V_{1}-V_{f}\right) \frac{1}{x L s+x R} \\
& I_{f}=I_{1}-I_{2} \\
& I_{2}=\left(V_{f}-V_{2}\right) \frac{1}{(1-x) L s+(1-x) R} \\
& V_{f}=\left(L_{f} s+R_{f}\right) I_{f} \\
& I_{C 2}=I_{2}-I_{L} \\
& V_{L}=\frac{1}{(1-x) C s} I_{C 2} \\
& I_{L}=V_{L} \frac{1}{L_{L} s+R_{L}}
\end{aligned}
$$

Using the above equations, a MATLAB/SIMULINK simulation model is made as shown in Fig. 3. The radial system is subjected to an Arc type fault at different locations from $x=0$ to full feeder length. For linear and nonlinear HIFs, the value of fault impedance $R f$ is given in the Appendix.

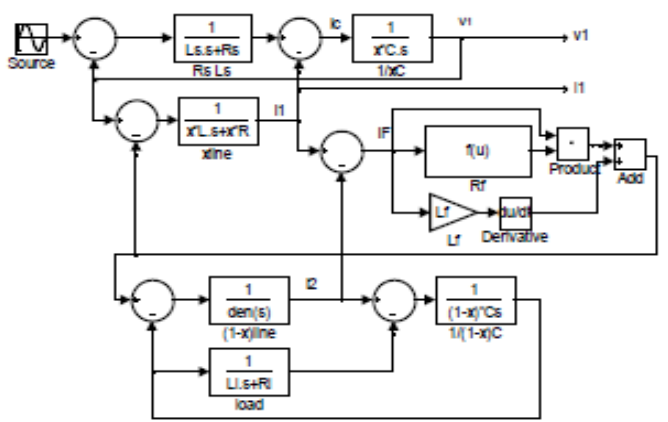

Fig.3. Matlab/Simulink functional model of radial system

Fault impedance $\mathrm{Rf}$ is given in the Appendix.

The Back-Propagation learning process works in small iterative steps: one of the example cases is applied to the network, and the network produces some output based on the current state of its synaptic weights (initially, the output will be random). This output is compared to the known-good output, and a mean-squared error signal is calculated. The error value is then propagated backwards through the network, and small changes are made to the weights in each layer. The weight changes are calculated to reduce the error signal. The whole process is repeated for each of the example cases, then back to the first case again, and so on. The cycle is repeated until the overall error value drops below some pre-determined threshold. At this point we say that the network has learned the problem "well enough" - the network will never exactly learn the ideal function, but rather it will generalized the ideal function.

\section{BACK-PROPAGATION ALGORITHM}

\section{Start with random weights}

2. Repeat

*For each example $e$ in the training set do

a. $O=$ neural net output; forward pass

b. $T=$ teacher output for $e$

c. Calculate error (T-O) at the output units

d. Computes $\Delta w$ for all weights from hidden layer to output layer; backward pass Continued

e. Computes $\Delta w$ for all weights from input layer to hidden layer; backward pass

Continued

$f$. Modify the weights in the network *End

3. Until classified correctly or stopping criterion satisfied

4. Return

\section{A. Feature extraction}

The radial distribution system is subjected to an arc type fault at different locations by varying $\mathrm{x}$, measured from the substation bus. The voltage and current signals at feeder terminals $v 1$ and $i l$ are used as detection signals as shown in Fig.4. The instantaneous values of these detection signals are captured and transformed into frequency domain using one cycle Fast Fourier Transform FFT. The FFT-harmonic vectors v3, i3, v5, and i5 are processed to obtain feature vectors and are used to train the network.

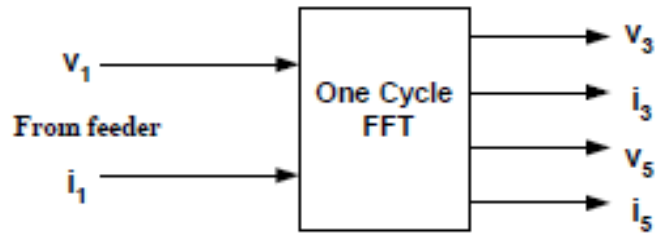

Fig.4 Feature vector extraction

Many cases of linear and nonlinear faults are simulated by varying the fault locations, source impedances and fault resistance. Equal numbers of linear and nonlinear fault cases are simulated. The obtained data is cast into a classification problem by associating half of the samples into linear and the other half into nonlinear cases.

\section{SIMULATION RESULTS}

The training and testing of the feed-forward neural network for distinguishing the linear and nonlinear faults is done using back propagation algorithm in metlab .The first step in training a feed-forward network is to create the network object .Before training a feed-forward network ,the weights and biases must be initialized. The newff command will automatically initialize the weights .The function sim simulates a network. Initially, reference vectors are randomly chosen for training the network. To 
International Journal of Advanced Research in Computer and Communication Engineering Vol. 3, Issue 10, October 2014

improve the characteristics of the network, the reference vectors are set to the locations matching the probability distribution of training vectors. This is done by dividing the training data into different classes and calculating the mean of the samples in these classes. Network structures with different number of reference vectors (subclasses) are tested. Fig. 5 shows the training error as a function of no. of epochs with random initial vectors. The data set is used to train a multilayer perceptron (MLP) with back propagation algorithm [7-8]. The architecture used for simulation consists of two hidden layers and one output layer. The output layer consists of one neuron whose output is zero or one for discrimination between linear and nonlinear faults. In the hidden layers, the neurons in the first hidden layer and second hidden layer can be varied to get optimum results. The transfer function in the neurons is log sigmoid because the output of this function ranges from 0 to 1 which matches the output of the linear fault cases ("1) and nonlinear fault cases ('0). The training algorithm used is the supervised Levemberg-Marquardt back propagation implemented in Mat lab's ANN toolbox. A training of $100 \%$ and testing of $94 \%$ was obtained when there were 8 neurons in the first hidden layer and 8 neurons in second hidden layer. But the algorithm took 46 epochs to reach this training.

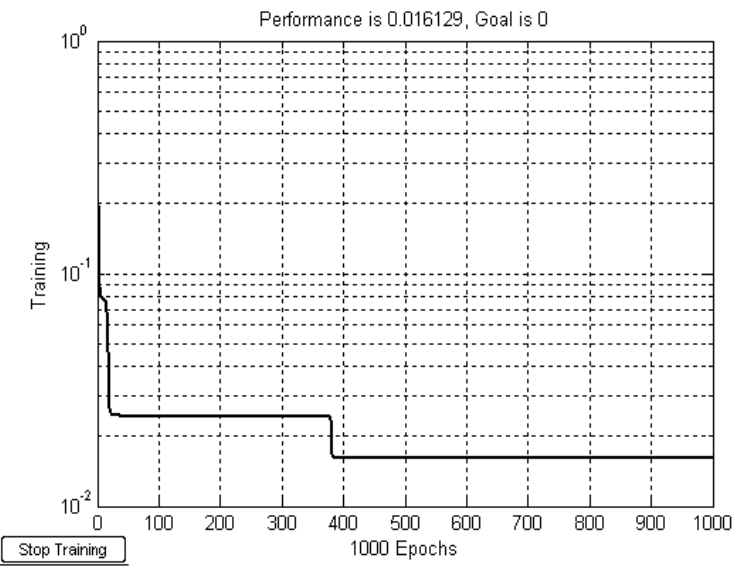

Fig.5 Training error as a function of number of epochs in case of MLP with back propagation algorithm.

\section{CONCLUSION}

A method for detecting the high impedance fault in a radial distribution system using Multilayer Perceptron networks is proposed in this paper. The proposed method is capable of detecting the arc-type high impedance faults with higher accuracy. The improvement in the performance of MLP networks is achieved by properly selecting the learning constant, architecture and setting the proper location of initial weight vectors.
(2) Nonlinear (Arc-type) HIF fault

$$
\begin{aligned}
& R f=R f 0+R f 1 \boldsymbol{\alpha}(\mathrm{i} f / \text { if } 0)^{\boldsymbol{\beta}} \\
& R f 0=20 \Omega, \quad R f 1=10 \text { to } 100 \Omega \\
& \boldsymbol{L} f=1 \text { to } 5 \mathrm{mH} \\
& \text { if } 0=70, \quad \alpha=0.6, \beta=2 .
\end{aligned}
$$

(c) Transmission line

$$
\text { Length } l=32 \mathrm{~km}
$$

$R=0.25 \Omega / \mathrm{km}, L=0.99472 \mathrm{mH} / \mathrm{km}$,

$C=0.01117 \mu F / \mathrm{km}$

(d) Load Parameters

$R l=180 \Omega, L l=0.2 H$

\section{REFERENCES}

[1] "High impedance fault detection technology,", Report of PSRC, Mar. 1996.

[2] A.R. Sedighi, M.R. Haghifam, O. P. Malik, and M.-H. Ghassemian, "High impedance fault detection based on wavelet transform and statistical pattern recognition," IEEE Transactions on Power Delivery, vol. 20, no. 4, pp. 2414-2421, Oct. 2005.

[3] M. Aucoin, "Status of high impedance fault detection," IEEE Transaction PAS, vol. 104, no. 3, pp. 638-644, Mar. 1985

[4] A. V. Mamishev, B. D. Russell, and C. L. Benner, "Analysis of high impedance faults using fractal techniques," in: IEEE Power Industry Computer Application Conf., pp. 401-406, 1995.

[5] C. J. Kim and B. D. Russell, "Classification of faults and switching events by inductive reasoning and expert system methodology," IEEE Transaction on. Power Delivery,vol.4,pp. 1631-1637, July, 1989.

[6] S. Ebron, D. L. Lubkeman, and M. White, "A neural network approach to the detection of incipient faults on power distribution feeders," IEEE Transactions on.Power Delivery,vol.5,pp.905914,April 1990

[7] L. A. Snider and Y. S. Yuen, "The artificial neural networks based relay for the detection of stochastic high impedance faults," Neurocomputing 23 , pp. 243-254,1998.

[8] A. M. Sharaf, L. A. Snider, and K. Debnath, "A neural network based relaying scheme for distribution system high impedance fault detection," in: Proceding. First New Zealand International TwoStream Conf. Artificial Neural Networks and Expert Systems,pp. 321-324,1993.

[9] B. M. Aucoin and B. D. Russell, "Detection of distribution high Impedance faults using burst noise signals near $60 \mathrm{~Hz}$," IEEE Transactions on. Power Delivery,vol. 2 , pp. 342-348,April 1987.

[10] D. I. Jeerings and J. R. Linders, "A practical protective relay for downconductor faults," IEEE Transactions on. Power Delivery. 6 ,pp. 565- 574,April 1991

[11] A.A. Girgas, W. Chang, and E. B. Makram, "Analysis of high impedance fault generated signals using a Kalman Filtering Approach," IEEE Transactions on. Power Delivery ,vol. 5, pp. 1714-1724,Oct.1990

[12] T. M. Lai, L. A. Snider, and E. Lo, "Wavelet transform based relay algorithm for detection of stochastic high impedance faults," Electrical Power System Research, 76 ,pp. 626-633,2006.

[13] A. E. Emanuel, D. Cyganski, J. A. Orr, S. Shiller, and E. M. Gulachenski, "High impedance fault arcing on sandy soil in $15 \mathrm{kV}$ distribution feeders:contributions to the evaluation of the low frequency spectrum," IEEE Transactions on. Power Delivery,vol.5, pp. 676-686,April 1990.

(a) AC System

\section{APPENDIX- 1}

$V S=(25 / \sqrt{3}) \sin (314 t), \quad R S=\mathbf{0 . 7 \Omega}$,

$\boldsymbol{L} S=7 m H$

(b) HIF Fault Model

(1) Linear HIF fault

$R f=30$ to $100 \Omega$

$$
\boldsymbol{L} f=1 \text { to } 5 \mathrm{mH}
$$

